\title{
El trabajo en pequeños grupos facilita la enseñanza-aprendizaje de Bioquímica
}

\author{
Work in small groups makes easy the \\ Biochemical teaching and learning
}

\author{
Gladis Magnarelli \\ Maria Martha Quintana ${ }^{\mathrm{I}}$ \\ Laura García ${ }^{\mathrm{I}}$ \\ Elena Villagrán De Rosso ${ }^{\mathrm{I}}$ \\ Luis Cabrera ${ }^{\mathrm{I}}$ \\ Lídia Ruiz-Moreno ${ }^{\mathrm{II}}$
}

PALABRAS CLAVE
- Educación Médica
- Bioquímica
- Enseñanza
- Evaluación educacional

\section{KEY WORDS}

- Medical Education

- Biochemistry

- Teaching

- Educational assessment
Recebido em: 17/12/2008 Reencaminhado em: 17/02/2009 Aprovado em: 11/03/2009
REVISTA BRASILEIRA DE EDUCAÇ̃̃O MÉDICA 33 (3) : $374-392 ; 2009$

\section{R E S U M E N}

El objetivo de este trabajo fue analizar la nueva propuesta de enseñanza de Bioquímica en la carrera de Medicina, que incluyó cambios en las estrategias de enseñanza-aprendizaje y de evaluación. Las clases expositivas fueron reemplazadas por la Discusión en Pequeños Grupos (DPG), en la que diez alumnos discuten situaciones del proceso salud-enfermedad. Los exámenes parciales individuales fueron reemplazados por la Evaluación en Pequeños Grupos (EPG) con la presentación de situaciones problema para la discusión y el análisis grupal. La información del presente estudio, de abordaje cualitativo, se obtuvo a partir de entrevistas semiestructuradas con docentes y alumnos. Los núcleos de análisis fueron: motivación, relaciones interpersonales, trabajo en grupo, construcción de conocimientos, comunicación, integración teórico-práctica y evaluación. La articulación de la DPG con las demás estrategias didácticas, como Clases de Consolidación, Seminarios Científicos, Trabajos Prácticos de Laboratorio y EPG motivó a docentes y alumnos, permitió la recuperación de conocimientos previos, la búsqueda activa de conocimientos e incentivó las relaciones interpersonales colaborativas y la integración conceptual. De esta forma, la incorporación de estrategias problematizadoras en grupos favoreció los procesos de aprendizaje significativo promoviendo las readecuaciones.

\section{A B S T R A C T}

The objective of this work was the analysis of the new biochemistry teaching proposal presented in the Medicine Career. This proposal included changes in the teaching-learning and evaluation strategies. Explanatory lessons were replaced for Discussions in Small Groups (DSG) where groups of ten students discuss different issues related to situations in the health-illness process. At the same time, individual mid-term exams were changed for Evaluations in Small Groups (ESG) with presentation of problematic situations for discussion and group analysis. The qualitative information contained herein was obtained from organized meetings with teachers and students. The data analysis was directed by: motivation, interpersonal relationships, group work, knowledge building, communication, theoretical and practical integration and evaluation. The DSG together with other didactic strategies of the department such as Consolidation Lessons, Scientific Seminars, Laboratory Written Assignments and ESG motivated teachers and students, allowed the recovery of previous knowledge and the active search for it, encouraged the interpersonal relationships and the conceptual integration. In this way, the inclusion of this type of strategies benefited the significant learning processes and promoted changes.

\footnotetext{
I Universidad Nacional del Comahue, Argentina.

II Universidad Federal de São Paulo, São Pauo, São Paulo, Brasil.
} 


\section{INTRODUCCIÓN}

La inserción de la asignatura de Bioquímica en el curso de Medicina tiene lugar generalmente en los primeros años y presenta características semejantes a otras materias básicas, tales como: gran volumen de información, dicotomía entre las estrategias de enseñanza teóricas y prácticas, escasa articulación con otras disciplinas del ciclo básico y con las del clínico, y poca vinculación con el perfil médico propuesto por la institución ${ }^{1}$. Además, el progreso de las investigaciones en Bioquímica proporciona un volumen de conocimientos que deben ser seleccionados y secuenciados en las actuales propuestas de enseñanza, junto con la aplicación de estrategias didácticas que favorezcan el desarrollo de competencias necesarias a los futuros médicos.

La educación universitaria para adecuarse a las necesidades actuales debe implementar propuestas curriculares innovadoras e impulsar en los estudiantes el compromiso con la promoción de acciones transformadoras, desarrollando la iniciativa y autonomía, la comunicación y trabajo en equipo, para permitirles interactuar con mayor eficacia en el ámbito personal, laboral y social.

Las actividades didácticas de carácter transmisivo, competitivas, centralizadas en el profesor, representan mayor esfuerzo docente y menos aprendizaje de los alumnos ${ }^{2}$. Es preciso promover la participación activa y solidaria, la habilidad de seleccionar, organizar y valorar la información científica, proporcionando un enfoque contextualizado de la Bioquímica y no una simple enumeración de datos fragmentados. En este contexto el grado y la calidad del aprendizaje de los alumnos dependen, en buena medida, de la mediación del docente para orientar procesos de aprendizaje significativo ${ }^{3}$.

Las propuestas programáticas constructivistas que privilegien el trabajo en grupo parecen favorecer los vínculos entre los diferentes actores del proceso de enseñanza-aprendizaje y con el objeto de conocimiento, superando la relación unidireccional entre profesores y alumnos ${ }^{4}$. Numerosos estudios confirman que las actividades que promueven una interacción entre los alumnos mejoran el aprendizaje al producir conflictos cognitivos entre individuos con opiniones divergentes que deben intercambiar ideas para llegar a un consenso $0^{5,6}$

Osorio $^{7}$ entiende por pequeños grupos aquellos en los cuales los individuos pueden reconocerse en su singularidad, manteniendo acciones interactivas en la búsqueda de objetivos compartidos. Así, el trabajo grupal representa una estrategia que posibilita al estudiante desarrollar competencias relacionadas con la expresión, interacción, habilidad para relacionarse con otros, considerando su integralidad ${ }^{8}$. Los métodos de aprendizaje cooperativos son importantes en el proceso de formación profesional ya que preparan ciudadanos más solidarios y comprometidos con los valores sociales ${ }^{9,10,11}$

\section{CONTEXTO Y OBJETIVOS DE LA INVESTIGACIÓN}

La presente investigación se llevó a cabo en la Escuela de Medicina de la Universidad Nacional del Comahue (UNCo), Argentina, en el marco de un Convenio de intercambio con el Centro de Desarrollo de la Enseñanza Superior en Salud (CEDESS) de la Universidad Federal de San Pablo (UNIFESP), Brasil.

La UNCo forma médicos generalistas desde su creación en 1998. Su propuesta curricular contempla tres ciclos: Biomédico, Clínico y de Síntesis de la Práctica Profesional. Este modelo curricular mantiene la estructura disciplinar pero incorpora actividades tutoriales semanales: los Talleres de Integración donde participan docentes de diferentes disciplinas utilizando la metodología de Aprendizaje Basado en Problemas (ABP). En estos Talleres de Integración, los temas de la asignatura Bioquímica, que se dicta en el primer cuatrimestre del primer año, se abordan junto con los de Genética, Biología Molecular e Histología.

La cátedra de Bioquímica, incluida en el ciclo Biomédico, cuenta con siete docentes rentados y tres docentes ad honorem, de los cuales seis tienen título de grado en Bioquímica y cuatro en Ciencias Biológicas. Entre los docentes hay uno con título de doctor, cuatro estudiantes de doctorado y dos de maestría. El cincuenta por ciento de los docentes participa en equipos de investigación básica mientras que el resto ejerce su actividad profesional en Bioquímica clínica. La Cátedra recibe cada año lectivo ciento veinte alumnos, número que se ha llegado a incrementar hasta 160 debido a los alumnos recursantes, por lo que la relación es de 1 docente cada 16 alumnos.

La propuesta programática desarrollada durante los primeros cuatro años de existencia de la asignatura incluía, entre otras estrategias, Clases Teóricas Expositivas y Exámenes Parciales individuales al final de cada módulo. La creciente inasistencia del alumnado, el escaso aprovechamiento del proceso de enseñanza-aprendizaje y el alto índice de reprobación motivaron una readecuación de la propuesta educativa. El presente trabajo tiene como objetivo analizar la nueva propuesta de enseñanza, implementada desde el año 2002, de la Asignatura Bioquímica en el Curso Médico de la Universidad Nacional del Comahue identificando limitaciones y posibilidades de la misma en base a la óptica de docentes y alumnos.

\section{PROCEDIMIENTOS METODOLÓGICOS}

En el presente estudio se utilizó un abordaje cualitativo ${ }^{12}$. Los procedimientos metodológicos comprendieron la recolección de datos a través de una entrevista semiestructurada a veinte alumnos que habían cursado y aprobado la asignatura de Bioquímica, 
y a cuatro docentes de la cátedra. Se obtuvo además información cuantitativa, sobre el porcentaje de alumnos aprobados a partir de los registros de evaluaciones.

El proceso de análisis de datos tuvo como referencia los siguientes núcleos orientadores: motivación, proceso de construcción de conocimientos (deducción de objetivos, formulación de hipótesis, elaboración de conclusiones), relaciones interpersonales, colaboración, comunicación, integración teórica-práctica y evaluación.

\section{Discusión en Pequeños Grupos: la experiencia vivencial de docentes y alumnos}

En la actual propuesta de enseñanza de Bioquímica, las Clases Teóricas Expositivas fueron reemplazadas por Discusión en Pequeños grupos (DPG) seguidas de Clases de Consolidación. Las estrategias didácticas, desarrolladas con frecuencia semanal, comprenden además: Resolución de Cuestionarios Guía, Trabajos Prácticos de Laboratorio y Seminarios Científicos, en las cuales se abordan los contenidos de cada módulo desde diferentes perspectivas.

En la DPG el docente presenta una situación de salud/enfermedad a un grupo de diez alumnos y los estimula a analizar diferentes aspectos, promoviendo el intercambio espontáneo de ideas y la definición de objetivos de aprendizaje. Un secretario anota los temas y preguntas surgidos, decisiones tomadas por mayoría y opiniones minoritarias relevantes. Al finalizar el proceso de discusión se elabora un resumen, que luego de llegar a un consenso, se entrega al docente. Esta actividad es limitada a 30 minutos de duración.

El equipo docente, posteriormente, registra los puntos principales surgidos en los diferentes grupos y selecciona los contenidos para lograr los objetivos planteados en cada módulo. Ese registro se utiliza para la planificación de la Clase de Consolidación, en la cual los grupos se reúnen y el docente presenta los objetivos y contenidos principales, hipótesis, acuerdos y desacuerdos surgidos en la DPG.

En el presente estudio se verificó que la motivación y participación de docentes y alumnos es estimulada, tanto en los momentos de planificación como durante la implementación de las actividades didácticas, ya que la selección de temas abordados en las clases de consolidación responde a las demandas, conocimientos previos y características del grupo. Así, los docentes y alumnos tienen un espacio de decisión en la planificación del proceso de enseñanza - aprendizaje ${ }^{13}$.

Los alumnos expresaron también su satisfacción en lo que se refiere a la motivación para la búsqueda activa de conocimiento y participación en las discusiones grupales con fundamento científico "[...] me di cuenta que tenia que leer más[...] que no sólo tenía que leer para el día de clase o para participar en el trabajo[...] sino leer para mí[...] para saber[...]"

El proceso mediante el cual se produce el aprendizaje significativo $^{14}$ requiere una intensa actividad por parte del alumno, que debe establecer relaciones entre el nuevo contenido y los elementos disponibles en su estructura cognoscitiva; juzgar y decidir la mayor pertinencia de estos, reformularlos, ampliarlos.

Sin embargo, la falta de preparación previa de los alumnos para la búsqueda activa de bibliografía fue notada por los docentes como una dificultad: "[...] los chicos no vienen con una formación desde el secundario para hacerse cargo de la búsqueda bibliográfica, les falta preparación[...]"

Desde todos los sectores se demanda una educación que responda a las exigencias de una sociedad compleja, contradictoria y cambiante. La enseñanza ligada a la transmisión de la información, que lleva a los aprendizajes de tipo asociativo, debe ser reemplazada por estrategias que generen procesos reflexivos ligados a la comprensión y la actitud crítica ${ }^{15}$. En tales procesos, la motivación, estado de tensión resultante de necesidades no satisfechas, desempeña un papel fundamental en el aprendizaje. Los estudiantes motivados trabajan enérgicamente y con intención. Cuando la motivación es intrínseca, el material aprendido proporciona su propia recompensa, la intención es comprender, el enfoque es profundo y el proceso consiste en relacionar las nuevas experiencias con los conocimientos previos

En cambio, cuando la motivación es extrínseca la retención, la comprensión, la transferencia son menos evidentes, las conductas estarán centradas en el miedo al fracaso o la necesidad de rendimiento. En este contexto la intención se limita a cumplir los requisitos de la tarea u obtener notas altas generando actitudes superficiales o adaptativas y los procesos cognitivos se reducen a memorizar la información en forma fragmentada y en asignar tiempos y esfuerzos según la rentabilidad ${ }^{16}$.

La formación de los futuros médicos no puede pretender que durante el período universitario se aborde toda la información existente, sino que se obtengan los elementos básicos que permitan comprender los problemas y situaciones de salud que el médico enfrenta en su práctica profesional, valiéndose de una base científica consistente.

En este sentido, la presentación y discusión de las situaciones problema utilizadas en la nueva propuesta promovieron el proceso de construcción de conocimientos científicos, que exigen niveles de competencias cognitivas más complejas, según se infiere de las siguientes expresiones de los docentes: “[...] A pesar que en las primeras DPG no tenían suficientes herramientas sobre Bioquímica, (los alumnos) podían elaborar hipótesis, algu- 
nas desacertadas, otras no tanto, plantearse sus objetivos y llegar a buen término. Salía casi natural[...]"

En coincidencia con lo expresado por los docentes, la DPG también fue valorizada por los alumnos como una instancia facilitadora del aprendizaje de contenidos científicos. Así, el objetivo de la DPG fue generar interés en el tema y estimular a los estudiantes a conocer más sobre la situación problema promoviendo el aprendizaje significativo. La intención es desarrollar en el alumno un pensamiento divergente, en el cual el docente no distribuye verdades sino que genera espacios de discusión ${ }^{17}$. Los estudios de Barbosa y Jófili ${ }^{9}$ muestran la conveniencia de abordar contenidos de Bioquímica, especialmente aquellos relacionados con el desarrollo del pensamiento científico, mediante estrategias de trabajo grupal cooperativo que promuevan la discusión entre individuos con opiniones divergentes que deben llegar a un consenso.

Dado que el clima emocional es de vital importancia en el proceso enseñanza-aprendizaje, se indagó a los alumnos si las actividades desarrolladas en pequeños grupos influyeron en las interacciones interpersonales docente/alumno y alumno/alumno: De las respuestas obtenidas, se infiere que estas relaciones fueron establecidas en un clima facilitador del aprendizaje: "[...]Los aspectos positivos, para mí, fueron que la cátedra nos ayudó, tuvieron consideración, tanto los docentes como los compañeros, te explicaban, aproveché el tiempo de clase y fue la (asignatura) que menos pensé en abandona[...]"

Para privilegiar el desarrollo de competencias como la intuición y el razonamiento, el docente debe actuar no como un mero intermediario entre la bibliografía y el educando sino como facilitador de la identificación de los problemas, ayudando al alumno a formularlos con claridad y a resolverlos convenientemente. “[...] ...los profesores de la cátedra nos ayudaban bastante, nos daban datos, para ambientarnos, para guiarnos a lo que queríamos llegar[...]"

En su práctica pedagógica es necesario que el docente promueva la discusión centralizada en el grupo, salvaguarde la libertad de expresión, facilite el diálogo de modo que todos puedan participar de la tarea colectiva designada y tenga sensibilidad para organizar las actividades de modo tal que faciliten el auto aprendizaje ${ }^{18,19}$.

Indagados sobre la experiencia de trabajar en grupos, los alumnos señalaron que, luego de una necesaria adaptación inicial, el clima emocional existente facilitó la colaboración y las interacciones personales: "[...] Aprendimos mucho de todos, había chicos que sabían bastante, aprendimos mucho de los profesores. Respecto del grupo, yo me sentí respetada."
Los docentes agregaron que la nueva propuesta educativa propició un acercamiento con los alumnos, que se volvieron más participativos y solidarios. En cuanto al trabajo grupal, la transformación del medio competitivo en ambiente cooperativo es un requisito importante para el adecuado desarrollo del proceso de enseñanza-aprendizaje $\mathrm{e}^{20,21}$

El desarrollo de competencias como la comunicación, abarcando la expresión de ideas, uso de vocabulario científico y la capacidad para saber escuchar y respetar las ideas de los demás, fue un aspecto desarrollado durante el trabajo grupal, según la perspectiva de los alumnos.

Los docentes observaron dificultades iníciales en la capacidad de expresión y en "escuchar al otro" y expresaron su compromiso con la necesidad de abordar esta cuestión como un contenido de aprendizaje y una competencia a ser desarrollada en los estudiantes. Es importante resaltar que un grupo no se constituye apenas como la suma de sus partes, sino que representa una red de relaciones y vínculos entre los integrantes, los que pasan a asumir determinados papeles sobre los cuales es necesario reflexionar con vistas a la formación integral de los alumnos. "[...]algunos alumnos quieren responder a todas las preguntas opacando el desempeño de otros mas tímidos que probablemente saben también la respuesta[...]Esto me parece ser un aspecto difícil de manejar."

Un aspecto positivo manifestado por un docente en cuanto a la dinámica grupal es el de permitir identificar alumnos con diferentes capacidades de expresión y comunicación y así poder estimular a aquellos menos familiarizados con la expresión oral.

Los docentes, indagados acerca de la integración de los conocimientos teórico-prácticos abordados en la DPG y en las diferentes estrategias didácticas implementadas en la cátedra, expresaron que esta relación se estableció de forma satisfactoria, tanto en las Clases de Consolidación y de Resolución de la Guía de Problemas como en los Trabajos Prácticos de Laboratorio, ya que los temas de cada módulo se abordaban en forma conjunta permitiendo la articulación conceptual en diferentes contextos.

Los profesores y estudiantes indagados sobre el aprovechamiento de otras actividades didácticas de la disciplina opinaron que existe una integración de la asignatura en su conjunto. Se destacó la función orientadora de la DPG para la actividad de Resolución de la Guía de Problemas, el desarrollo de los Trabajos Prácticos y Seminarios. La DPG contribuyó para el aprovechamiento de las Clases de Consolidación ya que la explicitación de dudas y expectativas de los alumnos propiciaba una mayor integración conceptual: “[...]las clases de consolidación son fundamentales[...] se presentan todas las hipótesis que fueron plantea- 
das en las DPG y se explicaba todo[...] todo lo que estaba relacionado hasta llegar a la hipótesis correcta[...] desde el inicio hasta el final[...] ayudaba a cerrar el tema."

Algunos alumnos manifestaron un aprovechamiento parcial de las Clases de Consolidación debido a problemas de infraestructura y al gran número de alumnos. Entre las sugerencias para mejorar el proceso de enseñanza aprendizaje, se incluyeron los referidos a la relación numérica docente - alumno, redistribución de carga horaria, mayor integración de contenidos en los Trabajos Prácticos de Laboratorio e incremento de actividades a ser realizadas en forma autónoma por los alumnos.

Los estudiantes citaron el proceso de adaptación al ritmo universitario, la inserción precoz de la disciplina en el curso médico y la falta de dedicación de parte de ellos como inconvenientes para el aprendizaje de Bioquímica.

Un aspecto relevante a ser destacado se refiere a la articulación, percibida por los alumnos, entre los contenidos de Bioquímica con otras asignaturas y la práctica médica, y al efecto movilizador de esa integración:

Es asombroso como se va relacionando con otras asignaturas, ahora vemos temas en Histología y los integramos con los de Bioquímica, o sea, lo que estoy estudiando ahora me va a servir para el futuro.

[...]yo había hablado con un médico y me había dicho que Bioquímica no servía para nada y a mí ahora me parece todo lo contrario, que la incluyo en todas las situaciones y eso me gustó.

Es necesario lograr la contextualización y revalorización del conocimiento que aporta la Bioquímica en la formación médica, relacionar los contenidos abordados con la práctica profesional superando la actual dicotomía entre los ciclos básico y clínico presentes en la mayoría de las escuelas médicas. En la opinión de Venturelli ${ }^{22}$ es preciso buscar la integración de conceptos y temas de las áreas biológica, psicológica y socioeconómica favoreciendo que las ciencias básicas permitan la comprensión real de los mecanismos íntimos de los procesos patológicos y situaciones de salud.

\section{Evaluación en Pequeños Grupos: posibilidades y desafíos}

El bajo rendimiento de los alumnos y las deficiencias para acompañar el proceso de enseñanza-aprendizaje, verificado en la propuesta programática anterior, motivaron el reemplazo de los Exámenes Parciales de múltiple opción, individuales, por la
Evaluación en Pequeños Grupos (EPG). El proceso de evaluación formativa fue complementado con evaluaciones escritas individuales, que incluyeron cuestiones abiertas y de múltiple opción, y con los registros docentes de la participación de los alumnos en las diferentes actividades didácticas, estructurados según criterios preestablecidos.

La EPG consiste en la presentación de una situación problema a un grupo de diez alumnos, en la que deben organizar sus conocimientos, interpretar, hacer generalizaciones, comunicar, justificar su respuesta y elaborar redes conceptuales. En esta actividad participan dos docentes, uno ejerce la función de coordinador mientras que el otro registra el proceso del grupo. Posteriormente se atribuye una nota individual en base a una guía de observación según criterios previamente acordados que abarcan las dimensiones cognitiva, procedimental y actitudinal.

Cuando se indagó a los docentes acerca del proceso de la EPG, expresaron que los alumnos, provenientes de un sistema educativo de carácter punitorio, se muestran retraídos al principio. No obstante, a medida que se desarrollan las actividades grupales los estudiantes gradualmente valorizan el aspecto formativo y colaborativo de esta modalidad de evaluación:

[...]En la primera evaluación los alumnos se sentían un poco inhibidos. Recién llegan de la escuela secundaria y no entendían bien lo que estaba sucediendo. Pero después pierden el miedo al aplazo, no tienen la presión, se relajan y pueden disfrutar un poco más, plantearse hipótesis y objetivos, y discutir entre ellos.

Los alumnos destacaron que la EPG contribuyó en el desarrollo de la habilidad de comunicación y uso adecuado del vocabulario científico, "[...]me corrigió para escuchar más y aprender del que está al lado y saber contestar lo que me preguntaban específicamente[...]". "Mi capacidad para defender mis ideas con sustento científico evolucionó[...]"

Hawort y Garrill ${ }^{23}$ sostienen que la comunicación verbal, competencia esencial de los futuros profesionales, recibe poca atención en la mayoría de los cursos de ciencias. Los autores encuentran una correlación positiva entre el desempeño de los alumnos en los exámenes escritos finales cuando participaron previamente de evaluaciones orales durante el curso, por lo que recomiendan su aplicación en el proceso de evaluación formativa.

En el presente estudio el análisis de las actas de examen de la asignatura reflejó un aumento en el número de alumnos que aprobaron la asignatura del 18, 21, 33 y 34 \% en los años posteriores a la implementación de la nueva propuesta. Los estudios de 
Anderson et al. ${ }^{21}$ también mostraron un mayor rendimiento en la evaluación de alumnos de bioquímica que realizaron estudio cooperativo en pequeños grupos, comparados con aquellos que participaron en experiencias tradicionales de enseñanza.

Sin embargo, la generalización y transferencia de conocimientos que se pretendió que los alumnos lleven a cabo en los exámenes escritos resultó un inconveniente para algunos de ellos ya que encontraban escasa relación entre los problemas planteados en la actividades didácticas y los temas indagados en las evaluaciones evidenciando una cierta dificultad en la transferencia de conocimientos a diferentes situaciones.

Cuando se indagó a los estudiantes sobre el papel de la EPG en la autoevaluación enfatizaron su utilidad para detectar dificultades, plantearse nuevas dudas y discutirlas en clase. Otro aspecto identificado por los alumnos en la autoevaluación incluyó la necesidad de desarrollar una mayor autonomía mediante el aprendizaje de técnicas de estudio que propicien el razonamiento y la integración de conceptos. Entendemos que, como lo describe Palou de Maté, (1998) ${ }^{17}$, es importante ayudar al alumno a generar una actitud de disconformidad cuando no comprende una explicación o un texto no le resulta claro. Esta ayuda no está referida a la sanción sino a señalar la importancia de las nuevas relaciones que proporciona un conocimiento trabajado y asumir una actitud de análisis de los factores que interfieren en su comprensión.

Pérez Gómez ${ }^{24}$ considera que una enseñanza de calidad es aquella que hace al alumno conocedor, responsable y capaz de intervenir sobre si mismo y su entorno físico y social. Esto evidencia no sólo la necesidad de nuevas formas de construcción de conocimientos que superen las prácticas tradicionales sino también que recuperen el valor de la evaluación pensando en la enseñanza y no sólo en la certificación y el control ${ }^{15}$. Por lo tanto, los resultados que se obtengan en la evaluación formativa deben ser útiles tanto para el profesor como para el alumno, ya que se relaciona directamente con el progreso de éste último y permiten reformular los objetivos de aprendizaje, enseñanza y currículo.

Según los datos obtenidos en el presente estudio, las EPG fueron de utilidad para detectar dificultades y realizar readecuaciones ya que el docente logró centrar su atención en comprender lo qué y cómo están aprendiendo sus alumnos a través del diálogo y la interacción, y no por medio de la descalificación, amenaza o castigo ${ }^{25}$. En este sentido, consideramos que ha contribuido el marco afectivo presente durante los encuentros, que permitió la libre expresión de los alumnos y la postura del profesor que evalúa no sólo para la acreditación ${ }^{25}$.
Algunas de las dificultades señaladas pueden ser reflejo de la necesidad de adecuar la formación pedagógica de los profesores a las demandas surgidas en el trabajo grupal. El profesor, por la propia naturaleza de su función, está convocado a ejercer la comprensión de las relaciones interpersonales y ser guía para la formación integral y el desarrollo de actitudes solidarias de los futuros profesionales.

En este sentido, implementar, analizar y compartir experiencias pedagógicas cooperativas constituye un eje central en el proceso de formación permanente de los profesores. La escuela y en particular la Escuela de Medicina constituyen un escenario inestimable para este aprendizaje.

\section{CONCLUSIONES}

La secuencia de actividades, donde la DPG introduce el tema y actúa como base para la planificación de las diferentes estrategias didácticas propuestas para módulo y la EPG funciona como síntesis integrativa, evidenció ser facilitadora del proceso de enseñanza aprendizaje y evaluación, según la perspectiva de docentes y alumnos que vivenciaron la experiencia.

Surgieron evidencias sobre la necesidad de una reorganización de las actividades didácticas en función del tiempo asignado al curso, revisión de las condiciones de infraestructura y recursos didácticos considerando el elevado número de alumnos y la mayor articulación entre las diferentes estrategias didácticas, especialmente de los trabajos prácticos de laboratorio. Participar en procesos de formación docente que posibiliten conocer las dinámicas grupales en lo que se refiere a interrelaciones, vínculos afectivos, liderazgos, percepción de límites, creatividad y capacidad de autonomía se sitúan también como desafíos para la práctica pedagógica.

Con relación al gran número de alumnos, característico de la mayoría de nuestras universidades, el trabajo en pequeños grupos ha evidenciado ser una estrategia que facilita la práctica docente ya que aumenta la motivación intrínseca y desarrolla la autonomía de los estudiantes. A su vez, el desarrollo de actitudes cooperativas entre los alumnos propicia el aprendizaje significativo de contenidos específicos al mismo tiempo que promueve la formación ética.

El trabajo en grupo se desarrolló en un ambiente emocional adecuado, respeto mutuo entre los diversos integrantes, favoreciendo instancias de construcción colectiva y colaborativa de conocimientos. La interacción docente/alumno y alumno/alumno, y con el objeto de estudio se vio estimulada, siendo destacada la función mediadora del docente. Asimismo, la integración y contextualización de los contenidos abordados con relación al curso médico y la práctica profesional fueron aspectos enfáticamente valorizados. 
La evaluación formativa se vio favorecida por el trabajo en pequeños grupos, tanto en la heteroevaluación como en la autoevaluación, con aumento del porcentaje de alumnos aprobados en la disciplina, desde la implantación de la nueva propuesta de enseñanza.

En este sentido concordamos con Osorio ${ }^{7}$ cuando nos señala que estar en grupos, trabajar en grupos, sentir y actuar en grupos es una praxis a la que nos conduce nuestra condición humana. Para esto, es necesario aprender a convivir de manera que los esfuerzos individuales se complementen en la búsqueda del bienestar colectivo, tarea en que el futuro médico debe estar comprometido ya durante su proceso de formación académica.

\section{REFERENCIAS}

1. Silva IF, Batista NA. Biochemistry in undergraduate health courses: structure and organization. Biochem Mol Biol Educ. 2003;31(6):397-401.

2. Luckie DB, Maleszewski J J, Loznak S D, Krha M. Infusion of collaborative inquiry throughout a biology curriculum increases student learning: a four-year study of "Teams and Streams". Adv Physiol Educ. 2004;28(4):199-209.

3. Novak JDE, Gowin DB. Aprender a aprender. Lisboa: Plátano Edições Técnicas; 1996.

4. Ruiz-Moreno L. Sonsogno MC, Batista SH, Batista NA. Mapa conceitual: ensaiando critérios de análise. Ciênc Educ (Bauru) [periódico na Internet]. 2007 [acesso em 2 nov.2007];13(3):453-63.Doi:10.1590/S1516-73132007000300012.

5. Vygotsky L. Pensamento e linguagem. São Paulo: Martins Fontes; 1988.

6. Silva SF, Beltrán-Núñez I. O ensino por problemas e trabalho experimental dos estudantes: reflexões teórico-metodológicas. Quím Nova [periódico na internet]. 2002 [acesso em 1 nov. 2007]; 25(6b). Doi: 10.1590/S0100-40422002000700023

7. Osorio L C. Psicologia Grupal: Uma nova disciplina para o Advento de uma era. Porto Alegre: Artmed; 2003.

8. Anastasiou LGC, Alves L P (Orgs.). Processos de Ensinagem na Universidade: Pressupostos para as estratégias de trabalho em aula. Joinville: Ed. da Univille; 2003.

9. Barbosa RMN, Jófili ZMS. Aprendizagem cooperativa e ensino de química: parceria que da certo. Ciênc Educ (Bauru). 2004;10(1):55-61

10. Fink LD. Beyond small groups: Harnessing the extraordinary power of learning teams. In: Michaelsen LK, Knight AB, Fink LD (Orgs.). Team-based learning: a transormative use of small groups. [monografia na internet] Sterling, VA: Stylus; 2004 [acesso em 26 nov. 2007]. Dispo- nível em: http://www.med.wright.edu/aa/facdev/_Files/PDFfiles/BeyondSmallGroups.pdf.

11. Slavin R E. Evidence-based reform: Advancing the education of student at risk. [documento na internet]. 2005 [acesso em 25 nov. 2007]. Disponível em: http:/ /www.americanprogress.org/projects / education/files/slavin_paper.pdf.

12. Minayo MCS. O desafio do conhecimento: pesquisa qualitativa em saúde. 9 $9^{a}$ ed. São Paulo: Hucitec; Abrasco; 2003.

13. Cellman S., in E.Litwin, A.Camilloni, S.Cellman, M.C Palou de Maté., S. Barco Eds La Evaluación de los aprendizajes en el debate didáctico contemporáneo, Paidos, Buenos Aires, 1998. pp. 35 - 66.

14. Novak J D. Understanding the learning process and effectiveness of teaching methods in the classroom, laboratory and field. Sci Educ. 1977;61(4):453-77.

15. Pastor L. in Palou de Maté MC, De Pascuale R, Herrera M, Pastor L Eds. Enseñar y Evaluar, Grupo Editor Multimedial S.R.L. Buenos Aires, 2001. p. 45-63.

16. Perrenaud P. Oficio do aluno e sentido do trabalho escolar. Porto; 1995.

17. Palou De Maté M C. in Litwin E, Camilloni A,Cellman S, Palou de Maté MC, Barco S. El Debate Didáctico Contemporáneo, Ed.Paidos, Buenos Aires, 1998. p. 93-132.

18. Romero R. Grupo, Objeto y Teoría. Buenos Aires: Lugar Editorial; 1987.

19. Cohen EG. Restructuring the classroom: conditions for productive small groups. Rev Educ Res. 1994;64(1):1-35.

20. Roger T, Johnson D W. Cooperative Learning: two heads learn better than one. In Context [periódico na internet]. 1988 [acesso em 7 out 2007]. Disponível em: http:/ / www.context.org/ICLIB/IC18/Johnson.htm

21. Anderson WL, Mitchell SM, Osgood MP. Comparasion of student performance in cooperative learning and traditional lecture-based biochemistry classes. Biochem Mol Biol Educ.2005;33(6):387-93.

22. Venturelli JE, Fiorini VML. Programas Educacionais Inovadores Em Escolas Médicas: Capacitação Docente. Rev Bras Educ Med. 2001;25(3):7-22.

23. Haworth IS, Garrill A. Assessment of verbal communication in science education. Biochem Mol Biol Educ. 2003;3(1):24-7

24. Pérez Gómez A. La cultura escolar en la sociedad neoliberal. Madrid:Morata; 1998. 
25. Herrera M.in Palou de Maté MC, De Pascuale R,Herrera M,Pastor L. Enseñar y Evaluar. Grupo Editor Multimedial S.R.L., Buenos Aires; 2001 p. 67 - 85.

\section{CONTRIBUCION DE LOS AUTORES}

Gladis Magnarelli participó de la búsqueda bibliográfica, del diseño del trabajo de investigación educativa, confección de entrevistas semi estructuradas, recopilación de datos sobre el rendimiento de los alumnos en la cursada, análisis y discusion de los resultados, redacción y revisión del artículo. Maria Martha Quintana participó de la búsqueda bibliográfica, confección de entrevistas semi estructuradas, entrevistas a los alumnos, descravación y registro de la información de las entrevistas, así como de la recopilación de datos sobre el rendimiento de los alumnos en la cursada, análisis y discusion de los resultados y revisión del artículo. Luis Cabrera, Elena Villagrán De Rosso y Laura García participaran de las entrevistas a los alumnos, descravación y registro de la información de las entrevistas, análisis y discusion de los resultados y revisión del artículo. Lidia Ruiz-Moreno de la búsqueda bibliográfica, del diseño del trabajo de investigación educativa, confección de entrevistas semi estructuradas, análisis y discusion de los resultados, redacción y revisión del artículo.

\section{CONFLICTO DE INTERESES}

Toda las fuentes de apoyo financeiro y material para la realización de este estudio fueron aportadas por la Escuela de Medicina, U.N. Comahue de Argentina y el de Centro de Desarrollo de la Enseñanza Superior en Salud, Universidad Federal de San Pablo, Brasil.

Además que no se ham estabelecido relaciones de carácer financiero ni de cualquer otro clase con ninguna organización o entidad que pudiera tener un conflicto de intereses respecto de algún aspecto del estudio realizado

\section{CORRESPONDENCIA}

Lídia Ruiz-Moreno

Centro de Desenvolvimento do Ensino Superior em Saúde

Universidade Federal de São Paulo

Rua Borges Lagoa. 1341 - Térreo

Vila Clementino - São Paulo

CEP.: 04038-034 SP

E-mail: ggmagnarelli@yahoo.com.ar

\section{R E S U O}

O objetivo de este trabalho foi analisar a nova proposta de ensino de Bioquímica na carreira de Medicina, incluindo as mudanças nas estratégias de ensino-aprendizagem e de avaliação. As aulas expositivas foram substituídas pela Discussão em Pequenos Grupos (DPG), em que dez alunos discutem situações do processo saúde-doença. As provas parciais individuais foram substituías pela Avaliação em Pequenos Grupos (APG) com a apresentação de situações problema para a discussão e a análise grupal. As informações para este estudo, de abordagem qualitativa, foram obtidas a partir de entrevistas semi-estruturadas com docentes e alunos. Os núcleos de análise foram: a motivação, as relações interpessoais, o trabalho em grupo, a construção de conhecimentos, a comunicação, a integração teórico-prática e a avaliação. A articulação da DPG com as demais estratégias didáticas, como as Aulas de Consolidação, Seminários Científicos, Trabalhos Práticos de Laboratório e APG motivou docentes e alunos, permitiu a recuperação de conhecimentos prévios, a busca ativa de conhecimentos e incentivou as relações interpessoais colaborativas e a integração conceitual. Desta forma, a incorporação de estratégias problematizadoras em grupos, tem favorecido os processos de aprendizagem significativa promovendo as readequações. 